Erkenntnisse über die gesundheitliche Basisversorgung und ihre infrastrukturellen Voraussetzungen seit einiger Zeit auch die Richtung der medizinischen Entwicklungszusammenarbeit der Direktion für Entwicklungszusammenarbeit und humanitäre Hilfe (DEH) des Eidgenössischen Politischen Departements und der grossen privaten Hilfsorganisationen in der Schweiz bestimmen. Es besteht heute kein Zweifel mehr darüber, dass medizinische Entwicklungshilfe eine völlige Abkehr von der bei uns geltenden Form der medizinischen Versorgung erfordert. Auch ist es - wenigstens im medizinischen Bereich - nicht mehr denkbar, dass Entwicklungszusammenarbeit in den Dienst eines materiellen oder anderen Profits für die Schweiz gestellt werden kann. Zum Beispiel ist ein massiver Einsatz junger Schweizer Ärzte in Entwicklungsländern als
Mittel zur Linderung des bei uns herrschenden Úberflusses an Assistenzärzten (wie er hie und da postuliert wird) im Rahmen des modernen Konzepts der gesundheitlichen Primärversorgung undenkbar. Anderseits wird ein gezielter Einsatz sozial- und präventivmedizinisch interessierter und gut vorbereiteter Mediziner bei der Verwirklichung dieses Konzepts in den am wenigsten entwickelten Regionen der Welt noch lange von grossem Nutzen sein. Zum bestmöglichen Einsatz der sich der Schweiz auf diesem Gebiet stellenden Möglichkeiten schiene es uns angebracht, den Kontakt und den Erfahrungsaustausch unter Ärzten und Organisationen, deren Interessen und Erfahrungen im Bereich der medizinischen Basisversorgung in der unterentwickelten Welt liegen, ganz besonders zu fördern.

\title{
Editorial
}

\section{Observations et réflexions sur la coopération au développement dans le domaine de la santé - Lettre de Colombie}

\author{
Th. Abelin ${ }^{1}$ et E. Zehnder ${ }^{2}$, actuellement à Cali, Colombie
}

Participer activement à la mise en place d'une infrastructure sanitaire dans un pays en voie de développement est le rêve de plus d'un médecin, d'une infirmière ou d'une laborantine. II y a peu de temps encore, l'idée que l'on se faisait du travail médical dans le tiers monde était celle du médecin «patriarche» travaillant en brousse ou en forêt, image idéalisée qui le montrait portant dans les villages lointains de l'Afrique, de l'Amérique du Sud ou de l'Asie les miracles de la médecine moderne. Pendant de longues heures, des malades innombrables qui s'étaient déplacés de loin attendaient d'être examinés et soignés par ce docteur venu de l'étranger, qui voyait son activité récompensée par des guérisons spectaculaires.

Cette activité trouvait sa justification dans l'aide directe apportée à des êtres humains souffrants, alors qu'on se posait moins de questions en ce qui

Adresses pour correspondance:

1 Prof. Dr méd., Institut de médecine sociale et préventive de l'Université de Berne, Hôpital de l' lle, CH-3010 Berne.

2 Dr méd., Apdo aéreo 20 300, Cali, Colombie (jusqu'en octobre 1979). concerne une structure sanitaire profitant à l'ensemble de la population. Ce n'est que vers le début des années soixante que des soucis d'ordre plus épidémiologique gagnèrent en importance: Combien d'habitants nécessitent des soins médicaux dans la province? Quelles sont les causes principales de morbidité et de mortalité? Quels sont les moyens en personnel et en matériel nécessaires pour garantir des soins, au sens conventionnel, à l'ensemble d'une région? Existe-t-il un système de soins, une modalité alternative, susceptible d'être étendu à toute la population et tenant suffisamment compte de sa situation économique et socio-culturelle?

Partant de ces interrogations, des projets expérimentaux très divers ont été entrepris. Ce sont principalement les modèles attribuant un rôle primordial au personnel auxiliaire et limitant l'offre à l'essentiel (traitement des maladies fréquentes - diarrhées, parasites, paludisme, etc. - et mesures de médecine sociale et préventive - vaccinations, assainissement, etc.) qui se sont révélés les plus efficaces. Depuis lors, certains pays du tiers monde (la Chine et la Tanzanie, par exemple) ont restructuré leur système sanitaire dans ce sens «révolutionnaire». 
L'occasion nous est offerte présentement de vivre de près cette évolution fascinante en tant que collaborateur-hôte de l'Institut de médecine sociale et préventive de l'Universidad del Valle, à Cali, Colombie. Cali est une métropole typique de l'Amérique du Sud, dont la population a augmenté d'environ $25 \%$ au cours des cinq dernières années pour atteindre 1240000 habitants. De nouveaux quartiers se créent sans cesse, ne consistant d'abord qu'en huttes de carton et de tôle et qui ne sont, au départ, pas raccordés aux réseaux d'alimentation en courant électrique et en eau, ni aux égouts. En même temps, Cali sert de champ d'expérience pour ce qui touche aux soins de santé primaires, depuis de nombreuses années.

En 1963 déjà, la Faculté de médecine de l'Universidad del Valle organisa, avec une aide extérieure, des prestations de soins dans une petite ville proche, action largement basée sur le concours de collaboratrices volontaires (Projet Candelaria). Par des enquêtes systématiques, des données précieuses furent récoltées sur l'état de santé de la population et sur les besoins de première nécessité des habitants. Des visites régulières à domicile par des «promotrices» avaient pour but de superviser la croissance des enfants, de procéder aux vaccinations habituelles, d'effectuer des traitements simples et de familiariser les parents avec les comportements les plus propices à la préservation de la santé. Un "passeport de santé» délivré à chaque mère de famille devait contribuer à intensifier son intérêts à l'évolution de la santé de ses enfants en bas âge. L'occasion était offerte aux étudiants en médecine d'apprendre à connaître, à l'hôpital de base, le rôle nouveau du médecin dans le cadre de soins de santé primaires restructurés.

En fonction des expériences acquises par le projet Candelaria, un nouveau système, basé sur la collaboration de promotrices travaillant à plein temps, a été mis sur pied par la suite dans un quartier de Cali comptant environ 60000 habitants (Projet Primops).

Un autre projet de recherche étudie le recours aux «promotrices» dans des conditions rurales (Projet Cimder), dans lequel les soins de santé ne représentent qu'une partie d'un programme de développement global. Une direction multidisciplinaire élabore la conception, surveille la réalisation et évalue les méthodes utilisées. Notons particulièrement certains des «instruments» développés par le projet et qui ont donné d'excellents résultats: un ruban de mesure tricolore (vert-orange-rouge) qui identifie le degré de sous-alimentation en mesurant la circonférence du bras, un appareil simple pour chlorer l'eau potable, une «pharmacie de village» disposant de six médicaments de base, un petit laboratoire, une cartothèque claire et maniable dans laquelle la promotrice inscrit les données relevées et les prestations effectuées dans chaque famille.

Pimar est le nom d'un projet mis en œuvre par deux médecins-chefs de petits hôpitaux, pour une population d'environ 90000 habitants défavorisés de Cali. Des visites à domicile régulières sont effectuées par du personnel auxiliaire, qui est chargé d'importantes fonctions de contrôle et de triage. On peut relever - entre autres - le club des hypertendus fondé par Pimar, qui compte quelque 400 membres.

Nous avons été particulièrement intéressés par les résultats d'un projet de recherche dans le domaine de la psychiatrie. Un groupe de malades psychiques choisis au hasard a été pris en charge dans son milieu de vie naturel par du personnel auxiliaire bien préparé. Ces collaborateurs «laïques» faisaient des visites quotidiennes au domicile de leurs malades, les aidant à résoudre les problèmes qui pouvaient se poser, discutant avec la famille et surveillant la prise de médicaments, si nécessaire, sous supervision de spécialistes en psychiatrie. Les résultats obtenus ont été comparés avec ceux du système conventionnel (clinique et médecin spécialisés) et ont été trouvés tout à fait semblables. La comparaison coût-avantage des deux modalités s'est révélée particulièrement favorable au nouveau système.

A noter également l'activité d'un groupe interdisciplinaire (Projet Prides), qui s'est spécialisé dans l'évaluation d'institutions existantes du domaine de la santé publique (quant à leur adéquation et leur efficacité) et qui formule des propositions de réorganisation tenant compte des besoins principaux de la population et de l'utilisation rationnelle des ressources à disposition. L'idée nous est venue qu'un organisme de ce genre aurait également une importante tâche à remplir en Suisse.

Un autre projet (Programa de Nutrición) comporte un groupe de spécialistes universitaires divers et s'occupe de tous les aspects de la nutrition. Son activité est basée sur un modèle des causes de la sous-alimentation qui tient compte de facteurs médicaux, socio-culturels, agricoles et économiques. Sur la base d'indicateurs sélectionnés, des données sont récoltées dans tout le pays et sont mises à la disposition du Ministère du Plan, pour lui permettre d'évaluer et d'améliorer ses programmes de nutrition (Plan Pan) en cours. Un projet de recherche de ce même groupe a montré qu'une période d'allaitement courte et le recours précoce à l'alimentation artificielle sont corrélés avec la fréquence des diarrhées et des symptômes de malnutrition qui s'ensuivent. Sur la base de cette constatation, il a été décidé de lancer à Cali une grande campagne pour la promotion de l'allaitement maternel. 
Enfin, il convient de mentionner le Projet «Vivamos mejor», en cours depuis cinq ans et financé jusqu'à ce jour principalement par des fonds suisses. "Vivamos mejor» s'est fixé pour objectif de réaliser un programme d'éducation sanitaire dans les quartiers pauvres de la ville et d'apporter ainsi sa contribution à l'amélioration de la situation sanitaire. On peut noter l'heureuse conception de ses jardins d'enfants, qui accueillent des enfants sous-alimentés et délaissés d'âge préscolaire. Le but du programme, qui dure six mois, est la promotion de leur santé psychique et physique. En même temps, leurs mères bénéficient de cours sur les moyens simples d'éviter la malnutrition et de favoriser le développement psychomoteur des enfants. Ce modèle a rencontré un grand intérêt auprès des instances de l'Etat et il y a de bonnes chances qu'à la fin de la période expérimentale il trouve un champ d'application beaucoup plus étendu.

L'existence de ces nombreux projets ne doit cependant pas faire illusion: le fait est qu'une partie importante de la population de Cali n'a pas à sa disposition de système de santé valable. Et les expériences décrites n'auraient que peu de sens si l'on ne parvenait pas à faire bénéficier toute la population marginale des résultats obtenus. La phase expérimentale est maintenant terminée; le temps est venu de procéder à une application très large. D'entente avec les responsables des projets décrits, une conception des soins de santé primaires pour l'ensemble de la population pauvre de la ville (environ un demi-million de personnes) est actuellement en voie d'élaboration.

L'aide extérieure a joué un rôle plus ou moins important dans pratiquement tous les projets décrits. Aujourd'hui, ils sont financés de plus en plus par des fonds colombiens. Par ailleurs, la Universidad del Valle entretient depuis une bonne vingtaine d'années des relations étroites avec des universités et fondations nord-américaines. De nombreux Colombiens ont pu suivre une formation postgraduée à l'étranger, ce qui a permis de confier très tôt l'entière responsabilité des programmes à des nationaux.

L'exemple de Cali permet d'identifier les éléments et phases nécessaires au développement d'un système de santé pertinent. En premier lieu, il s'agit d'élaborer un concept rationnel, applicable à large échelle et supportable sur le plan économique. Ce concept doit être testé et constamment amélioré dans des projets pilotes bien évalués. Dans une phase ultérieure, il s'agira de se procurer les fonds et de recruter le personnel nécessaire et de le mettre à disposition de grands groupes de population. Alors que dans la phase expérimentale des fonds et des professionnels étrangers peuvent encore jouer un rôle capital, il est nécessaire ensuite d'assurer un financement et une direction technique indigènes et d'arriver à une totale intégration dans les structures existantes.

Il existe en Colombie - pays relativement typique de ce que l'on nomme le tiers monde - une structure sanitaire mise en place par l'Etat, disposant d'un réseau relativement dense d'hôpitaux, de facultés de médecine et d'autres centres de formation, et d'organisations s'occupant au niveau national de problèmes particuliers (nutrition, planning familial, prévention du cancer, par exemple). Mais une telle infrastructure fait presque totalement défaut dans les pays les plus pauvres, ce qu'on peut appeler le quart monde. D'une manière générale, il faut des années pour former les responsables locaux, mais cette formation ne peut être reçue sur place. Et l'expérience des dernières décennies a montré combien il est problématique de l'entreprendre en Amérique du Nord, en Europe ou en URSS. Le contact dans ces dernières régions avec un mode de vie et une culture tout à fait différents rend pratiquement impossible ensuite le retour au pays d'origine et à ses problèmes prioritaires. De meilleurs résultats sont obtenus si les ressortissants du quart monde peuvent être formés par des centres d'enseignement du tiers monde, dans un contexte socioculturel et des conditions pratiques bien plus proches de ceux dont ils sont familiers.

C'est avec satisfaction que l'on peut constater que les enseignements tirés des expériences des dernières années déterminent maintenant les orientations de l'activité médico-sanitaire des grands organismes d'entraide officiels et privés en Suisse. Il ne fait plus de doute que, dans cette coopération, il est nécessaire de se distancer complètement des modèles de prestations de soins qui prévalent dans nos pays. Il n'est par ailleurs plus pensable - du moins dans le domaine médical - que la coopération au développement puisse être mise au service d'un profit, matériel ou d'une autre nature, pour la Suisse. Par exemple, l'envoi massif de jeunes médecins suisses dans les pays en développement pour alléger la pléthore qui se fait sentir chez nous (solution parfois préconisée) est un contresens dans l'optique moderne des soins de santé primaires. Ce qui ne veut pas dire que les régions défavorisées de notre planète n'ont plus besoin de médecins et d'autres personnels de santé des pays industrialisés. Mais il est essentiel que ceux-ci s'engagent à ce travail convenablement préparés, dans une optique de santé publique, et en étant prêts à fonctionner dans le cadre de modèles nouveaux de prestations de soins. Compte tenu de ces développements, il nous paraît souhaitable de promouvoir tout spécialement les contacts et les échanges entre tous ceux dont les intérêts et les expériences touchent aux soins de santé de base dans les pays en développement. 